NBI-HE-94-20

March 1994

hep-th/9403150

\title{
A HIERARCHY OF SUPERSTRINGS
}

\author{
Fiorenzo Bastianelli \\ The Niels Bohr Institute, Blegdamsvej 17, DK-2100 Copenhagen Ø, Denmark
}

Nobuyoshi Ohtafi'?

NORDITA, Blegdamsvej 17, DK-2100 Copenhagen Ø, Denmark

Jens Lyng Petersen虫

The Niels Bohr Institute, Blegdamsvej 17, DK-2100 Copenhagen Ø, Denmark

\begin{abstract}
We construct a hierarchy of supersymmetric string theories by showing that the general $N$-extended superstrings may be viewed as a special class of the $(N+1)$-extended superstrings. As a side result, we find a twisted $(N+2)$ superconformal algebra realized in the $N$-extended string.

\footnotetext{
${ }^{1}$ e-mail address: fiorenzo@nbivax.nbi.dk
}

${ }^{2}$ e-mail address: ohta@nbivax.nbi.dk, ohta@fuji.wani.osaka-u.ac.jp

${ }^{3}$ Permanent address and address after April 1, 1994: Department of Physics, Osaka University, Machikaneyama-cho 1-16, Toyonaka, Osaka 560, Japan

${ }^{4}$ e-mail address: jenslyng@nbivax.nbi.dk
\end{abstract}


Recently a remarkable discovery has been made that string theories may be interpreted as kinds of spontaneously broken phases of those with higher world-sheet symmetries [面]. In particular, it has been shown that the $N=0(N=1)$ strings can be viewed as a special class of vacua for the $N=1(N=2)$ superstrings [1-5]. It has also been shown that $N=2$ superstrings can be regarded as different phases of $N=4$ strings [6]. In this process it has been observed that a similar structure exists beyond $N=2$ [6, [], and it has been speculated that it is possible to embed the general $N$ superstrings into the $(N+1)$ superstrings for $N \geq 2$ [8, 1, 2].

The purpose of this paper is to realize explicitly the $N$-extended superstrings as special choices of the vacua in the $(N+1)$-extended superstrings, and to give a simple and explicit proof that this $(N+1)$ formulation is equivalent to the $N$ superstring. By $N$-extended superstrings we mean those based on the linear algebras found in ref. [9]. Our results apply to general $N$ superstrings for $N \geq 2$, and hence imply that there is an infinite hierarchy of superstrings. We also find a twisted $(N+2)$ superconformal symmetry realized in the $N$ string.

We will use the $N$-extended superspace to describe these theories. Our conventions follow closely those of ref. [10]: $Z=\left(z, \theta^{i}\right)$ with $i=1,2, \ldots, N$ denotes the $N$-extended super-coordinates and

$$
\begin{aligned}
D_{i} & \equiv \partial_{\theta^{i}}+\theta^{i} \partial_{z}, \quad\left\{D_{i}, D_{j}\right\}=2 \delta_{i j} \partial_{z} \\
\theta_{12}^{i} & \equiv \theta_{1}^{i}-\theta_{2}^{i}, \quad z_{12} \equiv z_{1}-z_{2}-\theta_{1}^{i} \theta_{2}^{i} \\
\theta^{N} & \equiv \frac{1}{N !} \epsilon^{j_{1} \cdots j_{N}} \theta^{j_{1}} \cdots \theta^{j_{N}}, \quad \theta^{N-i} \equiv \frac{1}{(N-1) !} \epsilon^{j_{1} \cdots j_{N-1} i} \theta^{j_{1}} \cdots \theta^{j_{N-1}}
\end{aligned}
$$

The $N$ superconformal algebra (SCA) is generated by the super stress tensor $T$, which satisfies the operator product expansion (OPE)

$$
T\left(Z_{1}\right) T\left(Z_{2}\right) \sim \frac{\theta^{N}}{z^{2}}\left(2-\frac{N}{2}\right) T\left(Z_{2}\right)+\frac{\theta^{N-i}}{z} \frac{1}{2} D_{i} T\left(Z_{2}\right)+\frac{\theta^{N}}{z} \partial T\left(Z_{2}\right),
$$

where we have used the shorthand notation $z \equiv z_{12}$ and $\theta^{N} \equiv \theta_{12}^{N}$. Central extensions can appear for $N \leq 4$ [10]. However, we will mainly focus on the cases which do not have central extensions $(N \geq 5)$, or have a vanishing value for the critical central charges 
$(N=3,4)$. We will comment on the case $N=2$ later on. One can construct a string theory by using the $N$ SCA as the (chiral) constraint algebra defining the states of the string. This information is encoded in a nilpotent BRST operator, constructed as the superspace integral of the BRST current $Q_{N}$,

$$
Q_{N}=C_{t}\left(T+\frac{1}{2} T\left(B_{t}, C_{t}\right)\right)
$$

where the reparametrization ghosts $\left(B_{t}, C_{t}\right)$ are fields of spin $\left(2-\frac{N}{2},-1\right), C_{t}$ being anticommuting for any $N$, and $T(B, C)$ denotes the super stress tensor of an arbitrary $B C$ system

$$
T(B, C)=(-1)^{N\left(\epsilon_{C}+1\right)}\left(-\lambda_{B} B \partial C+\lambda_{C} \partial B C+\frac{(-1)^{\epsilon_{B}}}{2} D_{i} B D_{i} C\right) .
$$

In this last formula, $\lambda$ and $\epsilon$ denote the conformal spin and Grassmann character of a field (even $\epsilon$ for bosonic fields and odd $\epsilon$ for fermionic ones), respectively, and satisfy

$$
\lambda_{B}+\lambda_{C}=1-\frac{N}{2}, \quad \epsilon_{B}+\epsilon_{C}=N .
$$

We also use the following correlator for a $\mathrm{BC}$ system

$$
C\left(Z_{1}\right) B\left(Z_{2}\right) \sim \frac{\theta_{12}^{N}}{z_{12}}
$$

The OPE of the BRST currents with itself can be computed and is given by

$$
Q_{N}\left(Z_{1}\right) Q_{N}\left(Z_{2}\right) \sim-\frac{(-1)^{N}}{4} \frac{\theta^{N}}{z} D_{i}\left(\left(D_{i} C_{t}\right) Q_{N}\right)\left(Z_{2}\right) .
$$

However, one can improve the BRST current by a total derivative term

$$
\tilde{Q}_{N}=Q_{N}-\frac{1}{4} D_{i}\left(\left(D_{i} C_{t}\right) B_{t} C_{t}\right)
$$

to make it completely nilpotent

$$
\tilde{Q}_{N}\left(Z_{1}\right) \tilde{Q}_{N}\left(Z_{2}\right) \sim 0
$$

This result will be helpful in constructing the general embedding to be discussed shortly and, more generally, to uncover a twisted $(N+2) \mathrm{SCA}$ realized in the $N$ string. 
The $(N+1) \mathrm{SCA}$ in $N$ superfields is given by

$$
\begin{aligned}
T\left(Z_{1}\right) T\left(Z_{2}\right) & \sim \frac{\theta^{N}}{z^{2}}\left(2-\frac{N}{2}\right) T\left(Z_{2}\right)+\frac{\theta^{N-i}}{z} \frac{1}{2} D_{i} T\left(Z_{2}\right)+\frac{\theta^{N}}{z} \partial T\left(Z_{2}\right), \\
T\left(Z_{1}\right) G\left(Z_{2}\right) & \sim \frac{\theta^{N}}{z^{2}}\left(\frac{3}{2}-\frac{N}{2}\right) G\left(Z_{2}\right)+\frac{\theta^{N-i}}{z} \frac{1}{2} D_{i} G\left(Z_{2}\right)+\frac{\theta^{N}}{z} \partial G\left(Z_{2}\right), \\
G\left(Z_{1}\right) G\left(Z_{2}\right) & \sim \frac{\theta^{N}}{z} 2 T\left(Z_{2}\right) .
\end{aligned}
$$

This algebra can be written as in (2) using $(N+1)$ superfields and with the $(N+1)$ stress tensor defined in an obvious notation by $T_{N+1} \equiv G_{N} / 2+\theta_{N+1} T_{N}$. Again, all the information on physical states of the $(N+1)$ string theory is encoded in the BRST current for the $(N+1)$ algebra

$$
Q=C_{t} T+C_{g} G+C_{t}\left(\frac{1}{2} T\left(B_{t}, C_{t}\right)+T\left(B_{g}, C_{g}\right)\right)-C_{g}^{2} B_{t},
$$

where the anticommuting reparametrization ghosts $C_{t}$ and the commuting supersymmetry ghost $C_{g}$ have spins -1 and $-\frac{1}{2}$, respectively.

The embedding of the $N$ string into the $(N+1)$ string is achieved as follows. We first take an arbitrary matter background $T_{m}$ for the $N$ string which satisfies eq. (2). We then add to this a $B C$ system denoted by $(\eta, \xi)$, with $\xi$ anticommuting and with spin $-\frac{1}{2}$. The spin and statistics of $\eta$ follow then from eq. (阿). With these ingredients at hand, we can construct the following background for the $(N+1)$ string:

$$
\begin{aligned}
T & =T_{m}+T(\eta, \xi) \\
G & =\eta+\xi T_{m}+\left(\frac{N}{2}-1\right) \xi \eta \partial \xi+\frac{(-1)^{N}}{4} \eta\left(D_{i} \xi\right)^{2}-\frac{1}{2}\left(D_{i} \eta\right)\left(D_{i} \xi\right) \xi,
\end{aligned}
$$

where $T(\eta, \xi)$ is the super stress tensor for the $(\eta, \xi)$ system. One can show that these generators indeed satisfy the operator products given in eq. (10).

This construction is easy to understand. The supersymmetry generator $G$ has the structure $G \sim \eta+\tilde{Q}_{N}(\eta, \xi)$, where $\tilde{Q}_{N}(\eta, \xi)$ is the improved BRST current of eq. (8) with the reparametrization ghosts $\left(B_{t}, C_{t}\right)$ replaced by $(\eta, \xi)$. Since the improved BRST current is fully nilpotent, only the contractions between $\eta$ and $\tilde{Q}_{N}(\eta, \xi)$ are nonvanishing and generate the super stress tensor $T$, as can be imagined recalling the usual BRST 
algebra. Note, however, that the spin of the $(\eta, \xi)$ system gets modified to the values $\left(\frac{3}{2}-\frac{N}{2},-\frac{1}{2}\right)$ while $\left(B_{t}, C_{t}\right)$ had originally spin $\left(2-\frac{N}{2},-1\right)$. Actually, this construction can be extended to reveal a twisted $(N+2)$ algebra in the $N$ string. We will postpone the description of such an algebra to the end of the paper. Note also that one can think of $(\eta, \xi)$ as fields with the same quantum number of $\left(B_{g}, C_{g}\right)$ but opposite statistics, so that they will cancel each other through the BRST quartet mechanism.

The BRST current corresponding to the particular background just constructed is obtained by substituting eq. (12) into eq. (11)

$$
\begin{aligned}
Q_{N+1} & =C_{t}\left(T_{m}+T(\eta, \xi)\right) \\
& +C_{g}\left(\eta+\xi T_{m}+\left(\frac{N}{2}-1\right) \xi \eta \partial \xi+\frac{(-1)^{N}}{4} \eta\left(D_{i} \xi\right)^{2}-\frac{1}{2}\left(D_{i} \eta\right)\left(D_{i} \xi\right) \xi\right) \\
& +C_{t}\left(\frac{1}{2} T\left(B_{t}, C_{t}\right)+T\left(B_{g}, C_{g}\right)\right)-C_{g}^{2} B_{t} .
\end{aligned}
$$

To prove that this particular class of $(N+1)$ string theories is equivalent to the $N$ string, we perform a canonical transformation to map $Q_{N+1}$ onto $Q_{N}+Q_{t o p}$, where $Q_{N}$ was given in (3) and

$$
Q_{\text {top }}=C_{g} \eta
$$

gives the BRST charge for a trivial topological sector. This was the strategy already employed in refs. [3, 4, 5, 6], where a canonical transformation was used to map the $(N+1)$ supersymmetric formulation of the $N$ string onto the standard formulation for $N=0,1,2$. In particular, we follow ref. [4 which presented the canonical transformation factorized in three parts, each of which is of simpler construction and interpretation. The first canonical transformation we perform is generated by

$$
R_{1}=\oint B_{t} C_{g} \xi
$$

where $\oint$ denotes superspace integration. The property of $R_{1}$ is to make $T_{m}$ inert under the extra supersymmetry generated by $G$. We find that this transformation acts as follows:

$$
\begin{aligned}
Q_{N+1}^{\prime} \equiv e^{R_{1}} Q_{N+1} e^{-R_{1}} & =C_{t}\left(T_{m}+\frac{1}{2} T\left(B_{t}, C_{t}\right)+T\left(B_{g}, C_{g}\right)+T(\eta, \xi)\right) \\
& -C_{g} \xi T\left(B_{g}, C_{g}\right)+C_{g}\left(\eta+\frac{1}{2} \xi \eta \partial \xi+\frac{(-1)^{N}}{4} \eta\left(D_{i} \xi\right)^{2}\right)
\end{aligned}
$$


Here we consistently drop total derivative terms which may appear on the right hand side of this equation, since they will not affect the BRST charge. Next, we perform a second transformation generated by

$$
R_{2}=\oint\left[\left(\frac{N}{4}-\frac{1}{2}\right) B_{g} C_{g} \xi \partial \xi+\frac{1}{4} C_{g} \xi D_{i} \xi D_{i} B_{g}+\frac{1}{8}\left(\xi \eta-C_{g} B_{g}\right)\left(D_{i} \xi\right)^{2}\right]
$$

to simplify the BRST algebra in the $\left(B_{g}, C_{g}, \eta, \xi\right)$ topological sector. This casts the BRST charge into

$$
Q_{N+1}^{\prime \prime} \equiv e^{R_{2}} Q_{N+1}^{\prime} e^{-R_{2}}=C_{t}\left(T_{m}+\frac{1}{2} T\left(B_{t}, C_{t}\right)+T\left(B_{g}, C_{g}\right)+T(\eta, \xi)\right)+C_{g} \eta
$$

A final transformation generated by

$$
R_{3}=\oint\left[(-1)^{N} C_{t}\left(\left(\frac{3}{2}-\frac{N}{2}\right) B_{g} \partial \xi+\frac{1}{2} \partial B_{g} \xi-\frac{(-1)^{N}}{2} D_{i} B_{g} D_{i} \xi\right)\right]
$$

is used to decouple the BRST reparametrizations from the topological sector. It maps modulo total derivatives (18) onto $Q_{N}+Q_{t o p}$, as given in eqs. (3) and (14).

Obviously the BRST charges constructed out of $Q_{N}$ and $Q_{t o p}$ commute with each other and are nilpotent, giving separate conditions on the physical states. The topological charge $\oint Q_{t o p}$ imposes the condition that the fields $\left(B_{g}, C_{g}, \eta, \xi\right)$ fall into a quartet representation of the charge and decouple from the physical subspace, and we are left with the degrees of freedom of the $N$ superstring only. Thus the $(N+1)$ string propagating in the background described by eqs. (12) is equivalent to the $N$ string. Note also that our similarity transformations manifestly preserve the operator algebra.

For the embedding $N=2$ into $N=3$, one has to recall that there is a central extension $\sim c /\left(3 z^{2}\right)$ appearing on the right hand side of eq. (2) with the critical value $c=6$. However, this central charge is balanced by a contribution $c=-6$ due to the $(\eta, \xi)$ system. We have checked that our realization (12) as well as our canonical transformations work also in this case. Thus our construction is valid for arbitrary $N \geq 2$ and, when combined with the embeddings found in ref. [1], leads to the amazing result that there exists an infinite hierarchy of superstrings. 
We have also found that out of $T_{m}$ and $(\eta, \xi)$ one can construct an $(N+2)$ SCA:

$$
\begin{aligned}
T\left(Z_{1}\right) T\left(Z_{2}\right) & \sim \frac{\theta^{N}}{z^{2}}\left(2-\frac{N}{2}\right) T\left(Z_{2}\right)+\frac{\theta^{N-i}}{z} \frac{1}{2} D_{i} T\left(Z_{2}\right)+\frac{\theta^{N}}{z} \partial T\left(Z_{2}\right) \\
T\left(Z_{1}\right) G^{ \pm}\left(Z_{2}\right) & \sim \frac{\theta^{N}}{z^{2}}\left(\frac{3}{2}-\frac{N}{2}\right) G^{ \pm}\left(Z_{2}\right)+\frac{\theta^{N-i}}{z} \frac{1}{2} D_{i} G^{ \pm}\left(Z_{2}\right)+\frac{\theta^{N}}{z} \partial G^{ \pm}\left(Z_{2}\right), \\
T\left(Z_{1}\right) J\left(Z_{2}\right) & \sim \frac{\theta^{N}}{z^{2}}\left(1-\frac{N}{2}\right) J\left(Z_{2}\right)+\frac{\theta^{N-i}}{z} \frac{1}{2} D_{i} J\left(Z_{2}\right)+\frac{\theta^{N}}{z} \partial J\left(Z_{2}\right), \\
G^{+}\left(Z_{1}\right) G^{-}\left(Z_{2}\right) & \sim \frac{\theta^{N}}{z^{2}}\left(1-\frac{N}{2}\right) J\left(Z_{2}\right)+\frac{\theta^{N-i}}{z} \frac{1}{2} D_{i} J\left(Z_{2}\right)+\frac{\theta^{N}}{z}\left(T+\frac{1}{2} \partial J\right)\left(Z_{2}\right), \\
J\left(Z_{1}\right) G^{ \pm}\left(Z_{2}\right) & \sim \pm \frac{\theta^{N}}{z} G^{ \pm}\left(Z_{2}\right) .
\end{aligned}
$$

The realization is given by

$$
\begin{aligned}
T & =T_{m}+T(\eta, \xi) \\
G^{+} & =\eta \\
G^{-} & =\xi T_{m}+\left(\frac{N}{2}-1\right) \xi \eta \partial \xi+\frac{(-1)^{N}}{4} \eta\left(D_{i} \xi\right)^{2}-\frac{1}{2}\left(D_{i} \eta\right)\left(D_{i} \xi\right) \xi \\
J & =\eta \xi .
\end{aligned}
$$

This is the twisted $(N+2)$ SCA present in the $N$ string. In fact, the fields $(\eta, \xi)$ correspond to the ghosts $\left(B_{t}, C_{t}\right)$, but their stress tensor is twisted in order to shift the spins from the value $\left(2-\frac{N}{2},-1\right)$ to the value $\left(\frac{3}{2}-\frac{N}{2},-\frac{1}{2}\right)$. For $N=2$, this reduces to the special case known in the literature [11]. That such a construction was possible was suggested by Berkovits as reported in the first of refs. [11]. One can use it to construct a consistent background for the $(N+2)$ string, and by performing canonical transformations it is possible to show that this formulation is also equivalent to the $N$ string, as explicitly demonstrated in ref. [6] for the case $N=2$.

Concerning linear superalgebras, it remains to be seen if one can formulate the small $N=4$ superstring [9] with $S U(2)$ symmetry as a particular class of the large $N=4$ superstring and the general large $N=4$ superstrings depending on a free parameter $x$ [10, 12] as special backgrounds for the $N=5$ strings. Another line of investigation is to try to embed strings into $W$-strings. For recent advances in this latter topic, see refs. [13]. We hope to discuss these issues elsewhere. 


\section{Acknowledgements}

We would like to thank Nathan Berkovits for valuable suggestions. N. O. would also like to thank Paolo Di Vecchia for discussions, support and kind hospitality at NORDITA, where this work was done.

\section{References}

[1] N. Berkovits and C. Vafa, preprint HUTP-93/A031, KCL-TH-93-13, hep-th/9310170 (1993).

[2] J. M. Figueroa-O'Farrill, Phys. Lett. B321 (1994) 344, and QMW-PH-93-30, hepth/9312033 (1993).

[3] H. Ishikawa and M. Kato, preprint UT-Komaba/93-23, hep-th/9311139 (1993).

[4] F. Bastianelli, Phys. Lett. B322 (1994) 340.

[5] N. Ohta and J. L. Petersen, preprint NBI-HE-93-76, hep-th/9312187 (1993), Phys. Lett. B, to appear.

[6] F. Bastianelli, N. Ohta and J. L. Petersen, preprint NBI-HE-94-08, hep-th/9402042 (1994), Phys. Lett. B, to appear.

[7] F. Bastianelli and N. Ohta, preprint NBI-HE-94-10, hep-th/9402118 (1994).

[8] N. Berkovits, private communication.

[9] M. Ademollo et al., Phys. Lett. 62B (1976) 105; Nucl. Phys. B114 (1976) 297.

[10] K. Schoutens, Nucl. Phys. B295 (1988) 634.

[11] A. Giveon and M. Roček, Nucl. Phys. B400 (1993) 145;

J. Gomis and H. Suzuki, Phys. Lett. 278B (1992) 266. 
[12] A. Sevrin, W. Troost and A. Van Proeyen, Phys. Lett. B208 (1988) 447;

E. Ivanov, S. Krivonos and V. Leviant, Phys. Lett. B215 (1988) 689.

[13] N. Berkovits, M. Freeman and P. West, preprint KCL-TH-93-15, hep-th/9312013 (1993); H. Kunitomo, M. Sakaguchi and A. Tokura, preprint OU-HET-187, hepth/9403086 (1994). 